\title{
Journal of Dermatology Research
}

Letter to Editor

\section{Acral Cutaneous Manifestations during COVID-19 Outbreak}

\author{
Ramon Grimalt ${ }^{*}$, Mario Cutrone ${ }^{2}$ \\ ${ }^{1}$ Universitat Internacional de Catalunya, Barcelona, Spain \\ ${ }^{2}$ Ospedale dell ‘Angelo Mestre, Venice, Italy \\ *Corresponding Author: Ramon Grimalt, Universitat Internacional de Catalunya, Barcelona, Spain; \\ E-mail: rgrimalt@uic.es
}

Received Date: 13-10-2020; Accepted Date: 29-10-2020; Published Date: 06-11-2020

Copyright $^{\oplus} 2020$ by Grimalt $\mathrm{R}$, et al. All rights reserved. This is an open access article distributed under the terms of the Creative Commons Attribution License, which permits unrestricted use, distribution, and reproduction in any medium, provided the original author and source are credited.

\section{Letter to Editor}

We describe a patient with multiple cutaneous lesions resembling chilblains affecting her feet. She was one of 5 patients with these findings that we have seen during the last week, 4 of them in Venice, Italy, one of them in Barcelona, Spain.

A 15-year-old girl with no relevant clinical history presented with reddish asymptomatic spots on her feet. No one in her family nor social environment has been diagnosed by COVID-19 since confinement.

On examination, we observed the presence of chilblain-like vasculitical aspects such as microtrombotic-like reddish and purpuric macules (Fig. 1).

The girl had no symptoms associated with upper respiratory tract infections, nor myalgia, fever nor headache.

We performed fast serology PCR testing on the patient, which provided negative results.

Four other patients with similar skin findings (and no other symptoms) were seen at the Pediatric Dermatology unit in Venice, Italy. PCR swab tests in these patients were negative for COVID-19 in all cases

In the past week, we have seen 5 patients affected by a new dermatological manifestation, all coinciding with the outbreak of COVID-19 infection throughout the world. 
They were all asymptomatic adolescents from 12 to 16 years of age. But none of them had a positive family history for COVID-19 infection.

In most cases the cutaneous vasculitical like eruptions appear in very mild or totally asymptomatic forms of the disease and mostly in adolescents. It seems to appear at the end of the process. They appear on both hands and feet but predominately on feet. The tendency is for spontaneous resolution but we need more time to follow this up.

It is rather surprising that the PCR in all these patients was negative but, as onset occurred at the same time as the outbreak of COVID-19 it appears to suggest a clear relationships between the two.

Recently one patient with similar clinical findings was biopsied in Switzerland (personal communication) by Prof Daniel Hohl and showed a lividoid occlusive vasculopathy.

Other dermatological findings of COVID-19 infection include signs of small blood vessel occlusion. Also petechiae or tiny bruises, transient lividoid eruptions, acrocyanosis and pseudo chilblain lupus have been reported [1-4].

We need further cases, biopsies and serological studies to be able to better understand this extremely frequent phenomenon that related in time with the outbreak of COVID-19 but could be also cause by other viruses.

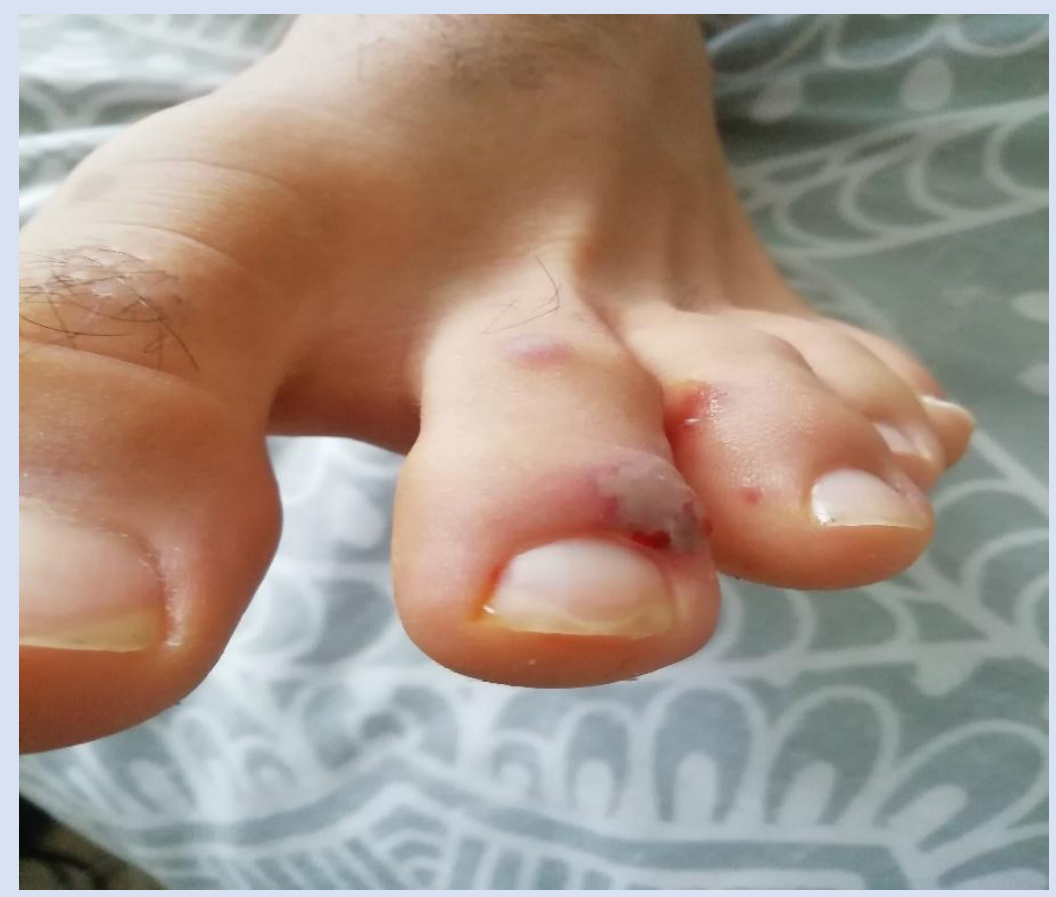

Figure 1: Hemorrhagic and petechial spots resembling chilblain in a 15-year-old girl. 


\section{Reference}

1. Qiu H, Wu J, Hong L, Luo Y, Song Q, Chen D. Clinical and epidemiological features of 36 children with coronavirus disease 2019 (COVID-19) in Zhejiang, China: an observational cohort study. Lancet Infect Dis. 2020.

2. Recalcati S. Cutaneous manifestations in COVID-19: a first perspective. J Eur Acad Dermatol Venereol. 2020.

3. Zhang Y, Cao W, Xiao M, Li YJ, Yang Y, Zhao J, et al. Clinical and coagulation characteristics in 7 patients with critical COVID-2019 pneumonia and acro-ischemia. Zhonghua Xue Ye Xue Za Zhi. 2020;41(4):302-7.

4. https://www.ejpd.com/en/mondayscase [Last Accessed on October 24, 2020]. 